\title{
Autoimmunity against hNinein, a human centrosomal protein, in patients with rheumatoid arthritis and systemic lupus erythematosus
}

\author{
SHEN-LONG HOWNG ${ }^{1,2^{*}}$, AN-KUO CHOU ${ }^{2,3^{*}}$, CHING-CHIH LIN ${ }^{4}$, ZHI-ANN LIN ${ }^{4}$, CHIH-JEN WANG ${ }^{1,2}$, \\ JOON-KHIM LOH ${ }^{1,2}$, ANN-SHUNG LIEU ${ }^{1,2}$, JENG-HSIEN YEN ${ }^{2,5}$, CHU-I LEE $^{6}$ and YI-REN HONG ${ }^{2,4}$ \\ ${ }^{1}$ Department of Neurosurgery, Kaohsiung Medical University Hospital, Kaohsiung; ${ }^{2}$ Graduate Institute of Medicine, \\ Kaohsiung Medical University, Kaohsiung 807; ${ }^{3}$ Department of Anesthesiology, Chang Gung Memorial Hospital, \\ Kaohsiung $833 ;{ }^{4}$ Department of Biochemistry, Faculty of Medicine, College of Medicine, Kaohsiung Medical University; \\ ${ }^{5}$ Division of Rheumatology, Department of Medicine, Kaohsiung Medical University Hospital; \\ ${ }^{6}$ Department of Medical Laboratory Science and Biotechnology, Fooyin University, Kaohsiung, Taiwan, R.O.C.
}

Received March 25, 2011; Accepted June 2, 2011

DOI: $10.3892 / \mathrm{mmr} .2011 .505$

\begin{abstract}
Centrosomes are organelles involved in the organization of the mitotic spindle and may also be the targets of autoantibodies in autoimmune diseases. Human Ninein (hNinein) is a centrosomal autoantigen that is identified by autoimmune patient sera. However, none of the hNineinspecific fragments recognized by the autoantibodies in rheumatoid arthritis (RA) and systemic lupus erythematosus (SLE) sera have been thoroughly characterized. We thus attempted to identify the fine specificity within the hNinein protein. In this study, four recombinant proteins in two isoforms of hNinein were used as autoantigens along with immunoassays as a molecular tool to investigate the prevalence of hNinein autoreactivity and its specificity in 22 RA and 32 SLE autoimmune disease sera. The data indicated a $50 \%$ higher prevalence of isoform $4 \mathrm{hNinein} \mathrm{N}$-terminal autoantibodies in RA sera, whereas $22 \%$ of SLE patients were autoreactive to the N-terminal of isoform $4 \mathrm{hNinein}$ compared to only a small percentage of autoreactive normal sera (5\%). These results showed that autoepitopes on autoantigen hNinein are restricted to the $\mathrm{N}$-terminal region and that a more significant proportion of RA patients exhibited centrosome reactivity.
\end{abstract}

Correspondence to: Dr Yi-Ren Hong, Department of Biochemistry, Faculty of Medicine, College of Medicine, Kaohsiung Medical University, 100 Shih-Chuan 1st Road, Kaohsiung 807, Taiwan, R.O.C.

E-mail: m835016@cc.kmu.edu.tw

Dr Chu-I Lee, Department of Medical Laboratory Science and Biotechnology, Fooyin University, 151 Chin-Hsueh Road, Ta-Liao Hsiang, Kaohsiung Hsien 83102, Taiwan, R.O.C.

E-mail:mt046@mail.fy.edu.tw

*Contributed equally

Key words: centrosomal proteins, hNinein, autoantigen, autoimmunity

\section{Introduction}

In mammalian cells, the large coiled-coil protein Ninein has been identified as a centrosomal protein (1). Ninein associates with the minus end of microtubules and is thought to play a significant role in the stabilization, positioning and anchorage of microtubules (2). Ninein is therefore essential for the centrosome to function as a microtubule-organizing center (MTOC) $(3,4)$. Previously, we identified a novel centrosomal protein, hNinein isoform 4 , using GSK-3 $\beta$ as bait in the yeast two-hybrid system, and observed that the full-length cDNA of hNinein consists of 2096 amino acids with a predicted molecular mass of $239 \mathrm{kDa}$. The features of this protein include a potential GTP binding site, a large coiled-coil domain together with four leucine zipper domains and a GSK-3 $\beta$ binding site (5). In the same report, it was also revealed that the hNinein genome corresponded to 29 exons of the genomic sequence on human chromosome 14q22. Additionally, an alternatively spliced isoform encoded a 2090 amino acid protein of $237.9 \mathrm{kDa}$, which was designated as hNinein-Lm (hNinein isoform 5). Comparing the amino acid sequence with that of hNinein, we found that hNinein-Lm exhibited an EF-hand $\mathrm{Ca}^{+2}$ binding domain in the N-terminus, which is similar to mouse Ninein. Northern blot analysis revealed that this hNinein isoform 5 was expressed more than the hNinein isoform 4 in the tissues examined (6). We thus postulated that the two different forms of Ninein are associated with a pair of centrioles in the centrosome structure $(1,2,7)$.

Antibodies directed against centrosomes have been described in a number of conditions including arthritis, Raynaud's phenomenon and systemic sclerosis (8-12). Although hNinein is associated with some autoimmune diseases $(13,14)$, none of the hNinein-specific fragments recognized by the autoantibodies in rheumatoid arthritis (RA) and systemic lupus erythematosus (SLE) sera have been fully characterized. We thus attempted to identify the fine specificity within the hNinein protein. In the present study, four recombinant proteins in two isoforms of human Ninein were constructed 
by the expression pattern as autoantigens and the autoreactivities of sera from patients with RA and SLE against the fusion protein of hNinein with His-tag were investigated by immunoassays. In our results, sera from patients were directed more specifically to the $\mathrm{N}$-terminal region but were not antigenic to the CCI region. Furthermore, the data indicated that a significant proportion of RA patients exhibited centrosome reactivity to the isoform $4 \mathrm{hNinein} \mathrm{N}$-terminal region. These data extend our understanding of the development of autoantibodies to the centrosomal protein hNinein and of the immunological aspects of RA.

\section{Materials and methods}

Patients. Sera were collected from 54 outpatients who received treatment at the Department of Immunity and Rheumatism at Kaohsiung Medical University (KMU, Kaohsiung, Taiwan). Out of 54 patients, 32 patients presented SLE and 22 presented RA. These diagnoses met the American College of Rheumatology (ACR) criteria for SLE and RA. Normal sera $(n=20)$ were collected from blood donors at KMU. The samples were obtained with informed consent, and the study was approved by the ethics committee of KMU.

Plasmid constructions, protein expression and purification. hNinein fragments (Fig. 1A) were amplified from plasmid DNA by PCR, cloned into pET-32a (Clontech). The generated plasmids were named pET-32a-NLM, NH, CCI and $\mathrm{CH}$. To determine the correctness of all the cloned sequences, nucleotide sequencing was performed by ABI PRISM ${ }^{\mathrm{TM}} 3730$ Genetic Analyzer (Perkin-Elmer). Plasmids pET-hNinein-NLM, pET-hNinein-NH, pET-hNinein-CCI and pET-hNinein- $\mathrm{CH}$, which express His-tagged $\mathrm{N}$-terminus-hNinein isoform 5 (corresponding to amino acids 1-471), His-tagged N-terminushNinein isoform 4 (corresponding to amino acids 1-477), His-tagged first leucine zipper-hNinein (corresponding to amino acids 455-1187 of hNinein isoform 5 and amino acids 461-1193 of hNinein isoform 4) and His-tagged C-terminushNinein (corresponding to amino acids 1616-1931 of hNinein isoform 5 and amino acids 1622-1937 of hNinein isoform 4, respectively) in Escherichia coli BL21/(DE3), respectively. To generate variant His-tagged hNinein fusion proteins, 0.31 of pET-32a/His-tagged-NLM, NH, CCI and CH-transformed Escherichia coli BL21 (DE3) was grown to the mid-log phase. Samples were induced at $37^{\circ} \mathrm{C}$ with isopropyl-1-thiob-D-galactopyranoside (IPTG), lysed by sonication in buffer A $(20 \mathrm{mM}$ Tris- $\mathrm{HCl}, 0.8 \% \mathrm{NaCl}, 0.1 \%$ lysozyme, and supplemented with protease inhibitors) and purified by chromatography on Ni-charged agarose. After washing, His-tagged hNinein fusion proteins were eluted with buffer containing $500 \mathrm{mM}$ imidazole.

Western blot analysis. For Western blot analysis, cells were harvested and washed once in phosphate-buffered saline (PBS). Cells were then resuspended in cell lysate buffer (20 mM PIPES, pH 7.2, $100 \mathrm{mM} \mathrm{NaCl,} 1 \mathrm{mM}$ PMSF, $1 \mathrm{mM}$ EDTA, $0.1 \%$ Chaps, and $10 \%$ sucrose). Samples were left for $30 \mathrm{~min}$ on ice, and centrifuged at $14,000 \mathrm{rpm}$ for $30 \mathrm{~min}$ at $4^{\circ} \mathrm{C}$. The supernatant was removed into a fresh centrifuge tube to which the protein sample buffer was added. The sample
A
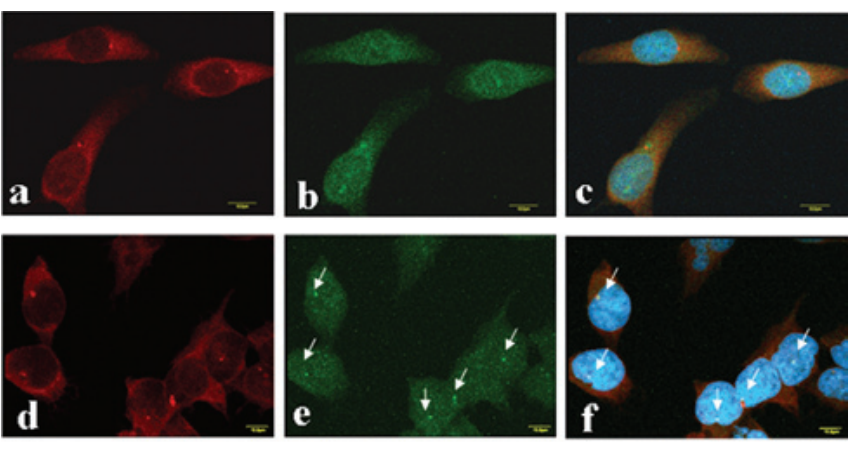

B
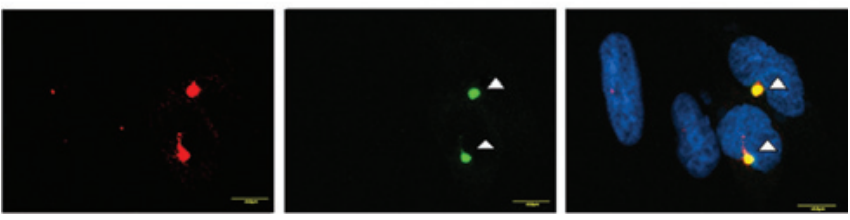

Figure 1. Immunofluorescent images of cells colabeled with hNinein and patient sera. (A) a, $\gamma$-tubulin staining of human cultured cells (HeLa) cells using a secondary rhodamine anti-human IgG (red); b, healthy sera on the same cell using a secondary fluorescein isothiocyanate (FITC) anti-human IgG antibody (green); c, an overlay of the a and b channels; $d, \gamma$-tubulin staining as in a; e, a representative RA serum on the same cell showing numerous 'nuclear speckles' (arrow) present using a secondary FITC antihuman IgG antibody (green); f, an overlay of the $\mathrm{d}$ and e channels showing a coincidence of centrosome and autoantibody staining (yellow), together with DNA staining to visualize the nucleus (blue). (B) The pattern of $\gamma$-tubulin staining (red) and localization of GFP-tagged hNinein (green) (arrowheads). The third panel is an overlay of the two left images revealing centrosome reactivity (yellow). Scale bars, $10 \mu \mathrm{m}$.

was then heated to $95^{\circ} \mathrm{C}$ for $5 \mathrm{~min}$ followed by analysis by $10 \%$ SDS-PAGE as previously described (15). Proteins were transferred to polyvinylidene difluoride membrane and incubated for $2 \mathrm{~h}$ in blocking buffer (5\% TBS/0.1\% Tween-20). His-tagged polyclonal antibodies or patient sera (1:250) were incubated in blocking buffer for $2 \mathrm{~h}$ at room temperature, and the membrane was incubated in horseradish peroxidaseconjugated anti-human IgG secondary antibody (Santa Cruz Biotechnology, Santa Cruz, CA, USA) for $1 \mathrm{~h}$. The protein bands were detected with diaminobenzidene substrate.

Enzyme-linked immunosorbent assay. Detection and titration of the antibody to a fragment of hNinein were examined using indirect enzyme-linked immunosorbent assay (ELISA). His-tagged hNinein fusion protein and His-tagged protein were prepared and used as antigens. Microtiter plates (96-well) were coated with His-tagged hNinein fusion protein in PBS (pH 7.4) for $15 \mathrm{~h}$ at $4^{\circ} \mathrm{C}$. His-tag protein was simultaneously coated in each well as a control. The wells were then washed with phosphate buffered saline plus 0.1\% Tween-20 (PBS-T), followed by treatment with $1 \%$ BSA in PBS for $2 \mathrm{~h}$ at room temperature. The wells were incubated with patient sera or the pooled negative control sera diluted at 1:250 with 1\% BSA in PBS for $2 \mathrm{~h}$ at $37^{\circ} \mathrm{C}$, followed by extensive rinsing with PBS-T. Subsequently, the wells were incubated with HRP-conjugated mouse anti-human IgG antibodyat a 1:2000 dilution for $1 \mathrm{~h}$ at $37^{\circ} \mathrm{C}$. The plates were washed with PBS-T, and a 100- $\mu$ l solution of o-phenylenediamine (Sigma, St. Louis, MO, USA) was added to each well. After $30 \mathrm{~min}$, the reaction was stopped by 
A
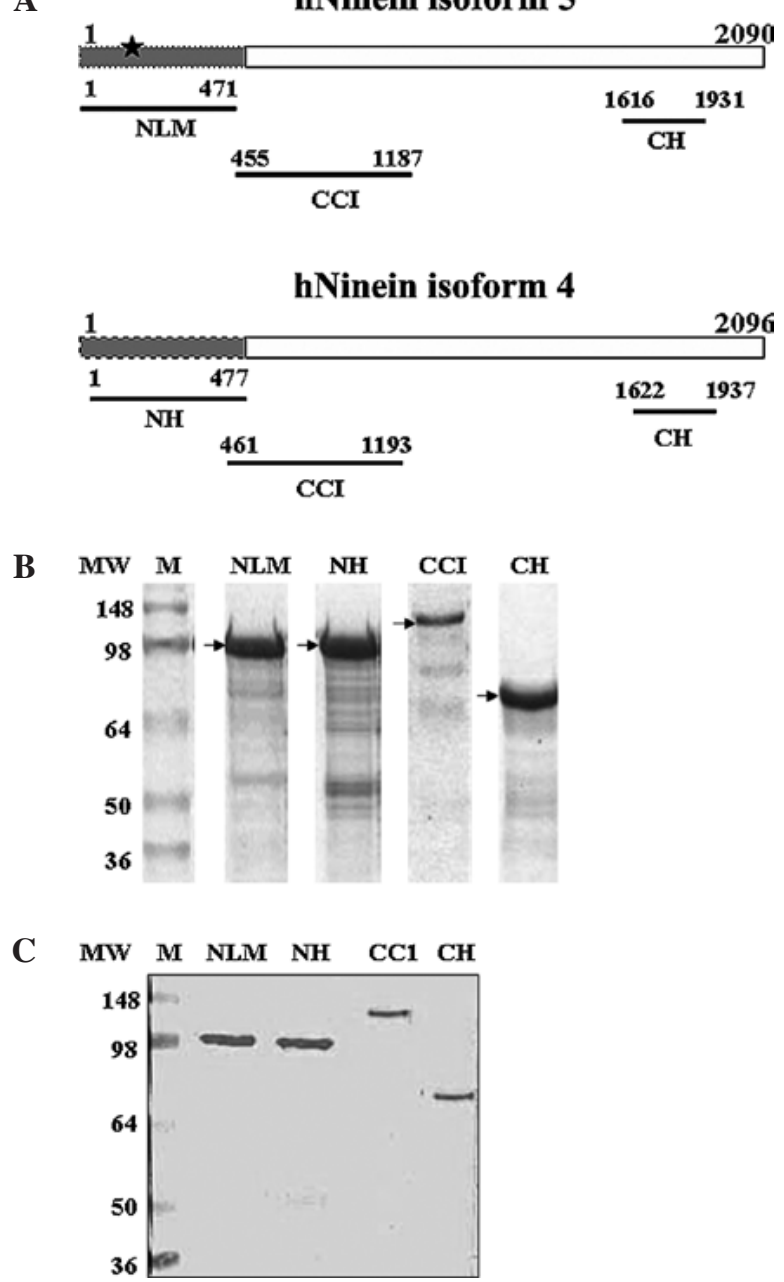

Figure 2. The distribution of autoantigenic regions on the hNinein autoantigen. (A) Schematic representation of the structures of two alternatively spliced human Ninein isoforms. The plot compares the amino acid sequence of hNinein isoforms 5 and 4. Open areas are identical regions that code the same peptide. Shaded areas are regions of different structure. Amino acid sequence comparison with hNinein showed that hNinein isoform 5 exhibited six fewer amino acids. Star indicates the $\mathrm{Ca}^{+2}$ binding site in hNinein isoform 5. (B) Coomassie blue staining of the partially purified pET-32a/Histagged partial Ninein proteins. ' $\mathrm{M}$ ' is the protein marker. The arrow indicates the encoded NLM, NH, CCI, and CH fusion proteins. (C) His-tagged NLM, $\mathrm{NH}, \mathrm{CCI}$ and $\mathrm{CH}$ fusion proteins were partially purified, separated on SDSPAGE, transferred to PVDF membranes and blotted with His-tag antibody.

adding $50 \mu \mathrm{l}$ of $3 \mathrm{M} \mathrm{H}_{2} \mathrm{SO}_{4}$. The specific corrected $\mathrm{OD}$ value of an individual sample was calculated by subtracting the optical density (OD) value of the His-tagged protein-coated well from that of the His-tagged hNinein fusion protein. Each sample was analyzed in duplicate and OD $490 \mathrm{~nm}$ was determined using a BioRad ELISA reader. The cut-off value designating a positive reaction was the mean OD of 20 normal sera +3 standard deviations (SDs).

Cell culture, transfection and indirect immunofluorescence. Human cultured cells (HeLa) were grown at $37^{\circ} \mathrm{C}$ in DMEM supplemented with $10 \%$ FCS and penicillin-streptomycin $(100 \mathrm{IU} / \mathrm{ml})$. For transient transfection studies, HeLa cells were seeded onto glass coverslips at a density of $1 \times 10^{6}$ cells per 12-well plate. DNA $(1 \mu \mathrm{g})$ was transfected into HeLa cells using Lipofectamine 2000 (Invitrogen). After 24 h, the cells
Table I. Clinical characteristics of RA patients whose sera reacted with hNinein autoantigens.

\begin{tabular}{lrrrrr}
\hline $\begin{array}{l}\text { Patient/ } \\
\text { age/gender }\end{array}$ & \multicolumn{1}{c}{$\begin{array}{c}\text { Criteria } \\
(16)\end{array}$} & NLM & NH & CCI & CH \\
\hline 1/59/F & $1,2,3,4,5,6,7$ & - & + & - & - \\
2/50/F & $1,2,3,4,5,6,7$ & + & + & - & + \\
3/60/F & $1,2,3,4,7$ & - & + & - & - \\
$4 / 28 / \mathrm{F}$ & $1,2,3,4,6,7$ & - & - & - & - \\
$5 / 43 / \mathrm{F}$ & $1,2,3,4,7$ & - & - & - & - \\
$6 / 67 / \mathrm{F}$ & $1,2,3,4,5,6,7$ & - & + & - & - \\
$7 / 65 / \mathrm{F}$ & $1,2,3,4,5,6,7$ & + & + & - & + \\
$8 / 61 / \mathrm{M}$ & $1,2,3,4,7$ & - & - & - & - \\
$9 / 69 / \mathrm{F}$ & $1,2,3,4,5,6,7$ & - & - & - & - \\
$10 / 66 / \mathrm{F}$ & $1,2,3,4,6,7$ & - & - & - & - \\
$11 / 52 / \mathrm{F}$ & $1,2,3,4$ & - & + & - & - \\
$12 / 64 / \mathrm{F}$ & $3,4,6,7$ & - & + & - & - \\
$13 / 49 / \mathrm{F}$ & $1,2,3,4,5,6,7$ & - & - & - & - \\
$14 / 72 / \mathrm{F}$ & $1,2,3,4,7$ & - & - & - & - \\
$15 / 62 / \mathrm{F}$ & $1,2,3,4,6,7$ & - & - & - & - \\
$16 / 60 / \mathrm{F}$ & $1,2,3,4,6,7$ & - & - & - & - \\
$17 / 59 / \mathrm{F}$ & $1,2,3,4,6,7$ & - & - & - & - \\
$18 / 72 / \mathrm{F}$ & $1,2,3,4,5,6,7$ & - & + & - & - \\
$19 / 83 / \mathrm{M}$ & $1,2,3,4,5,6,7$ & - & + & - & - \\
$20 / 78 / \mathrm{M}$ & $1,2,3,4,5,6,7$ & + & + & - & - \\
$21 / 71 / \mathrm{M}$ & $1,2,3,6$ & + & + & - & - \\
$22 / 63 / \mathrm{M}$ & $1,2,3,4$ & - & - & - & - \\
\hline & & & & &
\end{tabular}

1, Morning stiffness; 2 , arthritis of three or more joint areas; 3 , arthritis of hand joints; 4 , symmetric arthritis; 5 , rheumatoid nodules; 6 , serum rheumatoid factor and 7 , radiographic changes. The immunoreactivity of each fusion protein towards 22 rheumatoid arthritis (RA) sera in Western blot analysis is shown (+, immunopositive;-, immunonegative).

were fixed in cold methanol for $20 \mathrm{~min}$ and immunostained as previously described (16). The fixed cells were probed with human sera (1:250 dilution) and anti- $\gamma$-tubulin monoclonal antibody (1:500 dilution) (Sigma). The secondary antibodies were rhodamine-conjugated anti-human IgG, fluorescein isothiocyanate (FITC)-conjugated anti-human IgG $(1: 1,000$; Santa Cruz Biotechnology) and DNA was stained with DAPI (Roche). Coverslips were mounted with fluorescent mounting medium containing $15 \mathrm{mM} \mathrm{NaN}_{3}$. Images were captured using an OLYMPUS IX71 microscope (magnification, x100), 1.3NA UPlanFl objectives, and FLUOVIEW software (Universal Imaging). The z-axis stacks were compiled as single two-dimensional projections using FLUOVIEW software. The images were imported into Adobe Photoshop v5.0 for contrast manipulation.

\section{Results}

Analysis of centrosome reactivity by indirect immunofluorescence. We first investigated whether patient sera exhibited a specific Ninein staining pattern against centrosome structures, using indirect immunoflorecence to confirm the centrosome autoreactivity to hNinein antigens in vivo. In this test, the 
Table II. Clinical characteristics of SLE patients whose sera reacted with hNinein autoantigens.

\begin{tabular}{|c|c|c|c|c|c|c|}
\hline Patient/age/gender & Diagnosis & Criteria (18) & NLM & $\mathrm{NH}$ & $\mathrm{CCI}$ & $\mathrm{CH}$ \\
\hline $1 / 23 / \mathrm{F}$ & SLE & $1,3,4,5,9 \mathrm{~b}, 10 \mathrm{~b}, 10 \mathrm{c}, 10 \mathrm{~d}$ & - & + & - & - \\
\hline $2 / 54 / \mathrm{F}$ & SLE & $1,5,7 \mathrm{~b}, 8 \mathrm{a}, 8 \mathrm{~b}, 9 \mathrm{~d}, 10 \mathrm{~b}, 10 \mathrm{c}, 11$ & - & - & - & - \\
\hline $3 / 16 / \mathrm{F}$ & SLE & $2,5,6 a, 6 b, 7 a, 7 b, 9 a, 9 b, 9 d, 10 b, 10 c, 11$ & + & + & - & - \\
\hline $4 / 35 / \mathrm{F}$ & SLE & $5,10 \mathrm{~b}, 10 \mathrm{c}, 11$ & - & - & - & - \\
\hline $5 / 36 / \mathrm{F}$ & SLE & $5,7 \mathrm{a}, 9 \mathrm{~b}, 10 \mathrm{~b}, 10 \mathrm{c}, 11$ & - & - & - & - \\
\hline 6/39/F & SLE & $5,9 \mathrm{~d}, 10 \mathrm{~b}, 11$ & - & - & - & - \\
\hline $7 / 35 / \mathrm{M}$ & SLE & $7 \mathrm{a}, 7 \mathrm{~b}, 9 \mathrm{~d}, 10 \mathrm{~b}, 11$ & - & - & - & - \\
\hline $8 / 35 / F$ & SLE & $3,4,7 \mathrm{a}, 10 \mathrm{~b}, 11$ & - & - & - & - \\
\hline $9 / 23 / \mathrm{F}$ & SLE & $1,2,3,7 \mathrm{a}, 7 \mathrm{~b}, 10 \mathrm{~b}, 11$ & - & - & - & - \\
\hline $10 / 43 / \mathrm{F}$ & SLE & $7 \mathrm{a}, 7 \mathrm{~b}, 10 \mathrm{~b}, 11$ & - & - & - & - \\
\hline $11 / 64 / \mathrm{F}$ & SLE & $4,5,9 b, 10 b, 11$ & - & + & - & - \\
\hline $12 / 64 / \mathrm{F}$ & SLE & $3,7 \mathrm{a}, 10 \mathrm{~b}, 10 \mathrm{~d}, 11$ & - & - & - & - \\
\hline $13 / 26 / \mathrm{F}$ & SLE & $1,8 \mathrm{a}, 8 \mathrm{~b}, 9 \mathrm{a}, 9 \mathrm{~d}, 10 \mathrm{~b}, 10 \mathrm{~d}, 11$ & - & + & - & - \\
\hline $14 / 22 / \mathrm{F}$ & SLE & $4,5,9 \mathrm{~d}, 10 \mathrm{~b}, 11$ & - & - & - & - \\
\hline $15 / 22 / \mathrm{F}$ & SLE & $5,9 b, 10 b, 11$ & - & - & - & - \\
\hline $16 / 17 / \mathrm{F}$ & SLE & $1,2,3,4,5,9 b, 10 b, 11$ & - & - & - & - \\
\hline $17 / 36 / \mathrm{F}$ & SLE & $3,5,9 b, 9 d, 10 b, 11$ & - & - & - & - \\
\hline $18 / 51 / \mathrm{F}$ & SLE & $1,7 \mathrm{a}, 7 \mathrm{~b}, 9 \mathrm{~b}, 9 \mathrm{~d}, 10 \mathrm{~b}, 11$ & - & - & - & - \\
\hline 19/38/F & SLE & $1,3,7 a, 9 a, 9 b, 9 d, 10 a, 10 b, 10 c, 11$ & + & + & - & - \\
\hline 20/37/F & SLE & $1,7 a, 7 b, 9 a, 9 b, 9 d, 10 b, 11$ & - & - & - & - \\
\hline $21 / 25 / \mathrm{F}$ & SLE & $4,5,9 \mathrm{a}, 9 \mathrm{~b}, 9 \mathrm{c}, 9 \mathrm{~d}$ & - & - & - & - \\
\hline $22 / 31 / \mathrm{F}$ & SLE & $7 \mathrm{a}, 7 \mathrm{~b}, 8 \mathrm{a}, 8 \mathrm{~b}, 9 \mathrm{a}, 9 \mathrm{~b}, 9 \mathrm{c}, 9 \mathrm{~d}, 10 \mathrm{a}, 10 \mathrm{~b}, 10 \mathrm{c}, 10 \mathrm{~d}, 11$ & - & - & - & - \\
\hline 23/20/F & SLE & $1,3,5,7 \mathrm{a}, 7 \mathrm{~b}, 10 \mathrm{a}, 10 \mathrm{~b}, 10 \mathrm{c}, 10 \mathrm{~d}, 11$ & - & - & - & - \\
\hline $24 / 21 / \mathrm{F}$ & SLE & $5,7 \mathrm{a}, 9 \mathrm{~b}, 10 \mathrm{~b}, 11$ & - & - & - & - \\
\hline 25/33/F & SLE & $5,9 \mathrm{~b}, 9 \mathrm{~d}, 11$ & - & - & - & - \\
\hline 26/28/M & SLE & 9a, 9d, 10d, 11 & - & - & - & - \\
\hline 27/49/F & SLE & $1,5,10 \mathrm{a}, 10 \mathrm{~b}, 10 \mathrm{c}, 10 \mathrm{~d}, 11$ & - & + & - & - \\
\hline $28 / 23 / \mathrm{F}$ & SLE & $5,9 \mathrm{a}, 9 \mathrm{~b}, 9 \mathrm{c}, 9 \mathrm{~d}, 10 \mathrm{a}, 10 \mathrm{~b}, 10 \mathrm{c}, 10 \mathrm{~d}, 11$ & - & - & - & - \\
\hline 29/34/F & SLE & $3,5,9 \mathrm{~d}, 11$ & - & - & - & - \\
\hline $30 / 23 / \mathrm{F}$ & SLE & $5,7 \mathrm{a}, 9 \mathrm{~b}, 10 \mathrm{~b}, 11$ & - & - & - & - \\
\hline $31 / 26 / \mathrm{F}$ & SLE & $5,7 \mathrm{a}, 10 \mathrm{~b}, 11$ & - & - & - & - \\
\hline $32 / 22 / \mathrm{F}$ & SLE & $1,4,5,7 \mathrm{a}, 8 \mathrm{a}, 8 \mathrm{~b}, 10 \mathrm{~b}, 10 \mathrm{c}, 11$ & - & + & - & - \\
\hline
\end{tabular}

1, Malar rash; 2, discoid rash; 3, photosensitivity; 4, oral ulcers; 5, arthritis; 6, serositis (a, pleuritis; b, pericarditis), 7, renal disorder (a, persistent proteinuria in the urine; $b$, cellular casts); 8, Neurological disorder (a, seizures; b, psychosis); 9, haematological disorder (a, anemia; b, leukopenia; c, lymphopenia; d, thrombocytopenia); 10, immunological disorder (a, positive LE cell preparation; b, abnormal anti-DNA; c, Anti-Sm values; d, false postive VDRL); 11, abnormal ANA titer. The immunoreactivity of each fusion protein towards 32 systemic lupus erythematosus (SLE) sera in Western blot analysis is shown (+, immunopositive; -, immunonegative).

prevalence of hNinein autoreactivity was examined in patient sera or healthy control sera on human cultured cells (HeLa) using an antibody to $\gamma$-tubulin as a marker for the centrosome. The anti- $\gamma$-tubulin antibody gave a characteristic centrosome pattern of one or two small fluorescent dots which were either adjacent to or superimposed on the nucleus. Fig. 1A shows that a representative RA serum, but not a healthy control serum yielded an identical pattern without staining the nucleus or other cellular structures. The hNinein/GFP gene was then transfected to HeLa cells, and hNinein/GFP and $\gamma$-tubulin were stained in the same cells. Mounting evidence indicates that hNinein colocalized at the centrosome (Fig. 1B). This observation of the consistency between the hNinein autoantigen and hNinein/GFP suggests that the serum exhibited centrosomal hNinein autoreactivity.
Overexpression and purification of the four recombinant pET-32a/partial Ninein proteins. We then attempted to determine the antigenic regions of hNinein. For this purpose, four pET-32a plasmids with two isoforms of hNinein cDNA fragments were constructed by expression pattern as the peptides NLM, NH, CCI and $\mathrm{CH}$, which together largely covered the protein coding region of hNinein. The schematic structures of the two alternatively spliced human Ninein isoforms (isoforms 5 and 4) are shown in Fig. 2A. The partially purified recombinant NLM, NH, CCI and $\mathrm{CH}$ fusion proteins were produced as His-tagged fusion proteins and then blotted with His-Tag antibodies, as shown in Fig. 2B and C.

Detection of autoantibodies to hNinein in RA and SLE by Western blotting and ELISA. The percentage of those patients 


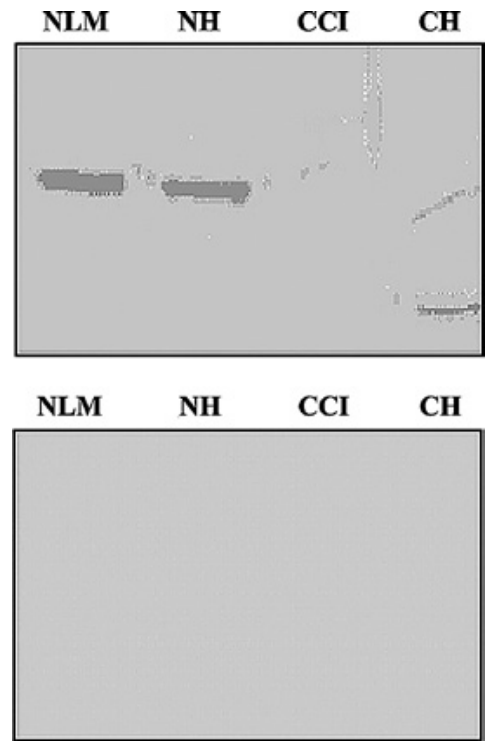

Figure 3. Representative Western blot using hNinein recombinant proteins to serum from patient RA-2 (top panel) and a normal healthy control (bottom panel).
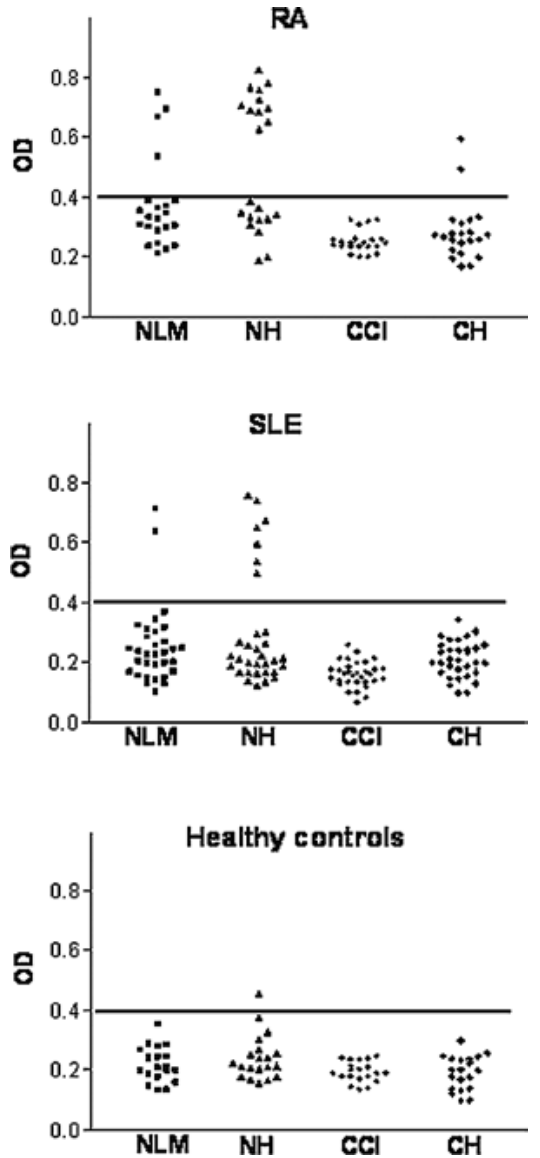

Figure 4. Autoantibody detection of hNinein NLM, NH, CCI and CH by ELISA. Top panel, 22 patients with RA; middle panel, 32 patients with SLE and bottom panel, 20 healthy controls. Bars indicate the cut-off value.

whose sera exhibited positive reactivity to hNinein NLM, NH, $\mathrm{CCI}$ and $\mathrm{CH}$ was then determined by immunoassay. Results from the Western blot assay revealed that hNinein fusions $\mathrm{NLM}, \mathrm{NH}, \mathrm{CCI}$ and $\mathrm{CH}$ were immunoreactive towards RA
Table III. Frequency of autoantibodies to specific hNinein autoantigens in autoimmune sera by Western blot and ELISA analysis.

\begin{tabular}{llccc}
\hline $\begin{array}{l}\text { Diagnosis } \\
\text { (no.) }\end{array}$ & \multicolumn{4}{c}{$\begin{array}{l}\text { Sera positive for hNinein autoantibodies } \\
\text { no. (\%) }\end{array}$} \\
\cline { 2 - 5 } & NLM & NH & CCI & CH \\
\hline RA (22) & $4(18)$ & $11(50)$ & $0(0)$ & $2(9)$ \\
SLE (32) & $2(6)$ & $7(22)$ & $0(0)$ & $0(0)$ \\
Normal (20) & $0(0)$ & $1(5)$ & $0(0)$ & $0(0)$
\end{tabular}

RA, rheumatoid arthritis; SLE, systemic lupus erythematosus.

and SLE antisera (Tables I and II). Fig. 3 shows Western blot results obtained with a representative sample of these sera. Notably, we found that the autoreactivity of test-positive sera shown as RA-2 was significantly strong to $\mathrm{NH}$ and NLM, weaker to $\mathrm{CH}$ and not reactive to CCI. A retrospective chart review showed that, there was no apparent correlation between serum autoantibody reactivity and clinical diagnosis. The specificity of NLM, NH, CCI and $\mathrm{CH}$ autoantibodies in sera from patients with RA and SLE was confirmed by ELISA, which is consistent with the data from the Western blot analysis using the same sera. Fig. 4 shows ELISA results obtained with 22 samples in RA, 32 samples with SLE and 20 samples from healthy donors. The number of positive sera and frequency of reactivity of all the patient sera are shown in Table III. In RA patient sera, we found that the frequency of autoreactivity against hNinein is NLM peptides $18 \%, \mathrm{NH}$ peptides 50\%, CCI peptides $0 \%$ and $\mathrm{CH}$ peptides $9 \%$. On the other hand, the frequency of autoreactivity against hNinein in SLE patient sera is NLM peptides $6 \%, \mathrm{NH}$ peptides $22 \%$, CCI peptides $0 \%$ and $\mathrm{CH}$ peptides $0 \%$. Only a small percentage of normal sera were autoreactive with the hNinein $\mathrm{NH}$ peptide (5\%). This result indicates that there is a hNinein-specific immune response in RA and SLE patients, particularly to epitope(s) in the NH fragment.

\section{Discussion}

Our main findings were first, hNinein autoantibodies were present more frequently in RA and SLE patients than in normal individuals; second, with the exception of CCI, each fragment of hNinein carries autoepitopes, among which $\mathrm{NH}$ was the most frequently recognized; third, autoantibodies to the C-terminal fragments of hNinein $(\mathrm{CCI}$ and $\mathrm{CH}$ ) were nonexistent or rare among the RA and SLE patients; and finally, the frequency of autoantibodies to $\mathrm{NH}$ was much higher than that of autoantibodies to NLM among both RA and SLE patients (17-19).

In this study, frequencies of autoantibodies to four specific hNinein autoantigens in a cohort of human sera were investigated by immunoassay. Our data revealed that the $\mathrm{N}$-terminal $\mathrm{NH}$ region was most frequently recognized in the serum of antihNinein antibody-positive patients. Specifically, $50 \%$ of the RA and $22 \%$ of the SLE patients who were positive for anti-hNinein 
antibodies reacted with the $\mathrm{NH}$ fragment. Of 18 anti-hNinein antibody-positive patients, 12 reacted only with $\mathrm{NH}$, and exclusively with NLM or CH. The remaining 6 anti-hNinein antibody-positive patients also reacted with NLM and/or CH, in addition to NH. In other words, the reactivity of NLM- and $\mathrm{CH}$-positive sera was always coexistent with $\mathrm{NH}$-positive reactivity. These data indicate that the $\mathrm{NH}$ fragment includes a specific immunodominant epitope of hNinein, and that antigens are being identified in the NLM and CH fragments.

hNinein is not reactive with autoantibodies due to its impermeability to the cell membrane. Therefore anti-Ninein antibodies have no direct effect on their target protein, hNinein. Our database search failed to detect sequence homology between the autoepitopes within the hNinein molecule and the sequences of any previously reported protein from a microorganism. However, the anti-hNinein antibodies may cross-react with some soluble antigens in the structure of sensorial joints as part of the joint destruction in RA or SLE. In addition, it is possible that the self-antigen $\mathrm{hNinein}$ would have to be released from injured tissues to invoke autoreactivity. The underlying reason for hNinein serving as an autoantigen remains to be determined. Furthermore, in our investigation, CCI exhibited no binding strength to hNinein, whereas the N-terminal fragments displayed the strongest binding strength. One possible explanation is that these autoepitopes in $\mathrm{NH}$ fragments may be recognized as surface structures on the centrosome involved in the formation of antigenic determinant regions, which may play unidentified functional roles as an important region for the induction of autoimmunity (20-22). The dominant coiledcoil structures of CCI fragments in dimer configuration are more capable of forming interactive linkages instead of epitope structure $(20,22)$.

Notably, although both the N-terminal hNinein isoforms are similar in structure, our data showed that the prevalence of the anti-NH autoantibody was significantly higher than that of the anti-NLM autoantibody in tested patients. Nevertheless, the finding of the N-terminal autoepitope on two isoforms of hNinein is consistent with the earlier report by Mack and coworkers (13) describing a large spectrum of Ninein autoepitope that recognized the recombinant fragment in a cohort of autoimmune sera. Thus, our data should provide important information concerning the role of the hNinein autoantigens within the centrosome complex and may support the notion that the specific immune response to a centrosome protein, such as hNinein, may be a crucial modulator of the pathologic process in this subset of autoimmune patients.

\section{Acknowledgements}

We thank Drs W.J. Tsai, J.R. Chen and C. Liu for providing sera and helpful comments on the manuscript. This study was supported by NSC 93-2320-B-037-037; NSC 93-2314B-037-015 and NHRI-EX99-9809SI (Taiwan) to YRH; KMUH98-8R02 to SLH.

\section{References}

1. Bouckson-Castaing V, Moudjou M, Ferguson DJ, Mucklow S, Belkaid Y, Milon G, et al: Molecular characterization of ninein, a new coiled-coil protein of the centrosome. J. Cell Sci 109: 179-190, 1996.
2. Mogensen MM, Malik A, Piel M, Bouckson-Castaing V and Bornens M: Microtubule minus-end anchorage at centrosomal and non-centrosomal sites: the role of ninein. J Cell Sci 113: 3013-3023, 2000.

3. Dammermann A and Merdes A: Assembly of centrosomal proteins and microtubule organization depends on PCM-1. J Cell Biol 159: 255-266, 2002.

4. Ou YY, Mack GJ, Zhang M and Rattner JB: CEP110 and ninein are located in a specific domain of the centrosome associated with centrosome maturation. J Cell Sci 115: 1825-1835, 2002.

5. Hong YR, Chen CH, Chang JH, Wang SK, Sy WD, Chou CK and Howng SL: Cloning and characterization of a novel human ninein protein that interacts with the glycogen synthase kinase 3beta. Biochim Biophys Acta 1492: 513-516, 2000.

6. Hong YR, Chen CH, Chuo MH, Liou SY and Howng SL: Genomic organization and molecular characterization of the human ninein gene. Biochem Biophys Res Commun 279: 989-995, 2000.

7. Lin CC, Cheng TS, Hsu CM, Wu CH, Chang LS, Shen ZS, Yeh HM, Chang LK, Howng SL and Hong YR: Characterization and functional aspects of human ninein isoforms that regulated by centrosomal targeting signals and evidence for docking sites to direct gamma-tubulin. Cell Cycle 5: 2517-2527, 2006.

8. Rattner JB, Mack GJ and Fritzler MJ: Autoantibodies to components of the mitotic apparatus. Mol Biol Rep 25: 143-155, 1998.

9. Gavanescu I, Pihan G, Halilovic E, Szomolanyi-Tsuda E, Welsh RM and Doxsey S: Mycoplasma infection induces a scleroderma-like centrosome autoantibody response in mice. Clin Exp Immunol 137: 288-297, 2004.

10. Gavanescu I, Vazquez-Abad D, McCauley J, Senecal JL and Doxsey S: Centrosome proteins: a major class of autoantigens in scleroderma. J Clin Immunol 19: 166-171, 1999.

11. Bao L, Varden CE, Zimmer WE and Balczon R: Localization of autoepitopes on the PCM-1 autoantigen using scleroderma sera with autoantibodies against the centrosome. Mol Biol Rep 25: 111-119, 1998.

12. Fritzler MJ, Zhang M, Stinton LM and Rattner JB: Spectrum of centrosome autoantibodies in childhood varicella and postvaricella acute cerebellar ataxia. BMC Pediatr 3: 11, 2003.

13. Mack GJ, Rees J, Sandblom O, Balczon R, Fritzler MJ and Rattner JB: Autoantibodies to a group of centrosomal proteins in human autoimmune sera reactive with the centrosome. Arthritis Rheum 41: 551-558, 1998.

14. Stillwell EE, Zhou J and Joshi HC: Human ninein is a centrosomal autoantigen recognized by CREST patient sera and plays a regulatory role in microtubule nucleation. Cell Cycle 3: 923-930, 2004.

15. Laemmli UK: Cleavage of structural proteins during the assembly of the head of bacteriophage T4. Nature 227: 680-685, 1970.

16. Krauss SW, Larabell CA, Lockett S, Gascard P, Penman S, Mohandas N and Chasis JA: Structural protein 4.1 in the nucleus of human cells: dynamic rearrangements during cell division. J Cell Biol 137: 275-289, 1997.

17. Conaghan PG, Green MJ and Emery P: Established rheumatoid arthritis. Baillieres Best Pract Res Clin Rheumatol 13: 561-575, 1999.

18. Tan EM, Cohen AS, Fries JF, Masi AT, McShane DJ, Rothfield NF, Schaller JG, Talal N and Winchester RJ: The 1982 revised criteria for the classification of systemic lupus erythematosus. Arthritis Rheum 25: 1271-1277, 1982.

19. Manzi S: Systemic lupus erythematosus: a model for atherogenesis? Rheumatology (Oxford) 39: 353-359, 2000.

20. Topfer F, Gordon T and McCluskey J: Intra- and intermolecular spreading of autoimmunity involving the nuclear self-antigens La (SS-B) and Ro (SS-A). Proc Natl Acad Sci USA 92: 875-879, 1995.

21. Rattner JB, Rees J, Whitehead CM, Casiano CA, Tan EM, Humbel RL, Conrad K and Fritzler MJ: High frequency of neoplasia in patients with autoantibodies to centromere protein CENP-F. Clin Invest Med 20: 308-319, 1997.

22. Mozo L, Gutiérrez C and Gómez J: Antibodies to mitotic spindle apparatus: clinical significance of NuMA and HsEg5 autoantibodies. J Clin Immunol 28: 285-290, 2008. 